\title{
The Integer Points on Three Related Elliptic Curves
}

\section{By Andrew Bremner and Patrick Morton}

\begin{abstract}
The integer points on the three elliptic curves $y^{2}=4 c x^{3}+13, c=1,3$, 9, are found, with an application to coding theory. It is also shown that there are precisely three nonisomorphic cubic extensions of the rationals with discriminant $-3^{5} \cdot 13$.
\end{abstract}

1. In [1] the Diophantine equation

$$
y^{2}=4 \cdot 3^{k}+13
$$

is shown to arise from coding theory, and its integer solutions are found. By considering congruence classes of $k$ modulo 3 , this equation gives rise to the three elliptic curves

$$
\begin{aligned}
& y^{2}=4 x^{3}+13, \\
& y^{2}=12 x^{3}+13, \\
& y^{2}=36 x^{3}+13 .
\end{aligned}
$$

We find here all integral solutions of (2), (3), (4), giving as a corollary all solutions to Eq. (1).

2. Since $Q(\sqrt{13})$ has class number 1, Eq. (2) immediately reduces to an equation

$$
\frac{y+\sqrt{13}}{2}=\varepsilon^{\kappa}\left(a+b \frac{1+\sqrt{13}}{2}\right)^{3},
$$

where $a, b \in \mathbf{Z}, \varepsilon=(3+\sqrt{13}) / 2$ is a fundamental unit of $Q(\sqrt{13})$, and where without loss of generality $\kappa=0, \pm 1$. Since $\alpha^{3} \in \mathbf{Z}[\sqrt{13}]$ for every integer $\alpha \in Q(\sqrt{13})$, the case $\kappa=0$ is impossible. Comparing coefficients of $\sqrt{13}$ in the two cases $\kappa= \pm 1$ gives respectively

$$
\begin{aligned}
& \kappa=1: 1=a^{3}+6 a^{2} b+15 a b^{2}+11 b^{3}, \\
& \kappa=-1: 1=a^{3}-3 a^{2} b+6 a b^{2}-b^{3} .
\end{aligned}
$$

Under the respective substitutions $(A, B)=(a+2 b, b),(A, B)=(a-b,-b)$ both (5) and (6) reduce to

$$
1=A^{3}+3 A B^{2}-3 B^{3} \text {. }
$$

We now work in $Q(\lambda)$, where $\lambda^{3}+3 \lambda-3=0$. It is straightforward to verify that the ring of integers in this field is $\mathbf{Z}[\lambda]$, and a fundamental unit is $\eta=1-\lambda$. (The

Received May 8, 1981; revised October 13, 1981

1980 Mathematics Subject Classification. Primary 10B10: Secondary 12A30. 
method of $[4$, p. 7] may be easily adapted to give a proof that $\eta$ is fundamental. See also [6].) Hence from (7), written as $\operatorname{Norm}(A-B \lambda)=1$, we deduce that

$$
A-B \lambda= \pm \eta^{n}
$$

for some integer $n$. Note that the minus sign cannot arise because Norm $\eta=1$.

Now $\eta=1-\lambda, \eta^{2}=1-2 \lambda+\lambda^{2}, \eta^{3}=1+3 \xi$, with $\xi=-1+\lambda^{2}$. If $n \equiv 2$ $(\bmod 3)$, then $\eta^{n} \equiv 1-2 \lambda+\lambda^{2}(\bmod 3)$, and $(8)$ gives an impossible congruence $(\bmod 3)$. Thus $n=3 N$ or $3 N+1$. If $n=3 N$, then we expand (8) in the form

$$
A-B \lambda=(1+3 \xi)^{N}=1+3 N \xi+3^{2}\left(\begin{array}{c}
N \\
2
\end{array}\right) \xi^{2}+\ldots
$$

Comparing coefficients of $\lambda^{2}$ in (9) gives

$$
0=3 N+3^{2}\left(\begin{array}{c}
N \\
2
\end{array}\right)(-5)+3^{3}\left(\begin{array}{c}
N \\
3
\end{array}\right)(\cdot)+\ldots
$$

If $3^{\nu} \| N$, then every term in this expansion except the first is divisible by $3^{\nu+2}$, giving a contradiction modulo $3^{\nu+2}$. Accordingly, $N=0$ is the only possibility, which does indeed give a solution $(A, B)=(1,0)$. Alternatively, we can invoke a result of Skolem [5] to show that (10) has at most one solution, which is thus $N=0$.

(See also [3, p. 54], and [7].)

Similarly, if $n=3 N+1$, we obtain

$$
\begin{aligned}
A-B \lambda & =(1-\lambda)(1+3 \xi)^{N} \\
& =1-\lambda+3(1-\lambda) N \xi+3^{2}(1-\lambda)\left(\begin{array}{c}
N \\
2
\end{array}\right) \xi^{2}+\ldots,
\end{aligned}
$$

and comparing coefficients of $\lambda^{2}$ gives

$$
0=3 N+3^{2}\left(\begin{array}{c}
N \\
2
\end{array}\right)(-8)+\ldots
$$

As before, $N=0$ is the only solution, corresponding to $(A, B)=(1,1)$.

The solutions $(1,0)$ and $(1,1)$ of $(7)$ give the solutions $(a, b)=(1,0),(-1,1)$ to $(5)$ and $(a, b)=(1,0),(0,-1)$ to $(6)$, which in turn give $(x, y)=(-1,3),(3,11),(-1,-3)$, $(3,-11)$ as the only solutions of $(2)$.

3. Equation (3) reduces to the equation

$$
\frac{y+\sqrt{13}}{2}=\varepsilon^{\kappa}(4+\sqrt{13})\left(a+b \frac{1+\sqrt{13}}{2}\right)^{3}, \quad \kappa=-2,-1,
$$

where we choose the sign of $y$ so that $y \equiv 1(\bmod 3)($ in order that $4+\sqrt{13}$ divide the left-hand side). Comparing coefficients of $\sqrt{13}$ we have

$$
\begin{aligned}
& \kappa=-2: 1=-a^{3}+6 a^{2} b-3 a b^{2}+5 b^{3}, \\
& \kappa=-1: 1=a^{3}+3 a^{2} b+12 a b^{2}+7 b^{3} .
\end{aligned}
$$

We write (11) in the form

$$
1=\operatorname{Norm}(A-B \theta),
$$

where $(A, B)=(-a+2 b, b)$ and $\theta^{3}-9 \theta+15=0$. The ring of integers in $Q(\theta)$ is $\mathbf{Z}[\theta]$, and a fundamental unit is $\rho=-53+18 \theta+9 \theta^{2}$, so from $\left(11^{\prime}\right)$ we deduce that

$$
A-B \boldsymbol{\theta}= \pm \rho^{n}, \quad n \in \mathbf{Z} \text {. }
$$


Setting $\rho=1+9 \xi$, with $\xi=-6+2 \theta+\theta^{2}$, and expanding 3-adically, we see by the same arguments as in Section 2 that $n=0$ is the only solution, giving $(a, b)=(-1,0)$ and $(x, y)=(1,-5)$.

Similarly, write (12) in the form

$$
1=\operatorname{Norm}(A-B \phi),
$$

where $(A, B)=(a+b, b)$ and $\phi^{3}+9 \phi-3=0$. The ring of integers in $Q(\phi)$ is $\mathbf{Z}[\phi]$, and a fundamental unit is $\delta=1-3 \phi$, with Norm $\delta=1$. From $A-B \phi=\delta^{n}$ we have the 3-adic expansion

$$
A-B \phi=1-3 n \phi+3^{2}\left(\begin{array}{l}
n \\
2
\end{array}\right) \phi^{2}-3^{3}\left(\begin{array}{l}
n \\
3
\end{array}\right) \phi^{3}+\ldots,
$$

and comparing coefficients of $\phi^{2}$ yields

$$
0=3^{2}\left(\begin{array}{l}
n \\
2
\end{array}\right)+3^{4}\left(\begin{array}{l}
n \\
4
\end{array}\right)(-9)+3^{5}\left(\begin{array}{l}
n \\
5
\end{array}\right)(\cdot)+\ldots .
$$

By Skolem [5] this has at most two solutions. But $n=0$ and $n=1$ do give solutions, and hence these are the only ones. (Note that elementary arguments will also succeed as before.) Thus $(a, b)=(1,0),(-2,3)$, leading to $(x, y)=(-1,1),(29,541)$.

4. Treating Eq. (4) in the same manner, we deduce first of all that

$$
\frac{y+\sqrt{13}}{2}=\varepsilon^{\kappa}(4+\sqrt{13})^{2}\left(a+b \frac{1+\sqrt{13}}{2}\right)^{3}, \quad \kappa=-2,-1,
$$

where $y \equiv 1(\bmod 3)$. Comparing coefficients gives the equations

$$
\begin{aligned}
& \kappa=-2: 1=a^{3}+12 a^{2} b+21 a b^{2}+19 b^{3}, \\
& \kappa=-1: 1=5 a^{3}+33 a^{2} b+78 a b^{2}+59 b^{3} .
\end{aligned}
$$

In fact (13) is

$$
1=\operatorname{Norm}\left((a+10 b)+b \phi^{2}\right)
$$

with $\phi$ defined as in $\left(12^{\prime}\right)$. Thus

$$
a+10 b+b \phi^{2}=\delta^{n}=1-3 n \phi+3^{2}\left(\begin{array}{l}
n \\
2
\end{array}\right) \phi^{2}-3^{3}\left(\begin{array}{l}
n \\
3
\end{array}\right) \phi^{3}+\ldots
$$

and comparing coefficients of $\phi$ yields the only solution $n=0$ as above, giving $(a, b)=(1,0)$ and $(x, y)=(1,7)$.

Further, it may be checked that the right-hand side of (14) is Norm $\Lambda$, where

$$
\Lambda=(-19 a-43 b)+(2 a-b) \theta+(2 a+3 b) \theta^{2},
$$

and $\theta$ is defined as in $\left(11^{\prime}\right)$. Thus $\Lambda= \pm \rho^{n}$, so that $\Lambda \equiv \pm 1(\bmod 3)$. However this gives the congruences modulo 3 :

$$
-19 a-43 b \equiv \pm 1, \quad 2 a-b \equiv 0, \quad 2 a+3 b \equiv 0,
$$

which are clearly incompatible. Hence (14) has no solutions and $(1, \pm 7)$ are the only integer points on (4).

5. To summarize, we have

THEOREM. The only integer points on

(i) $y^{2}=4 x^{3}+13$ are $(-1, \pm 3),(3, \pm 11)$;

(ii) $y^{2}=12 x^{3}+13$ are $(1, \pm 5),(-1, \pm 1),(29, \pm 541)$;

(iii) $y^{2}=36 x^{3}+13$ are $(1, \pm 7)$. 
COROLlaRY. The only integer solutions of

$$
y^{2}=4 \cdot 3^{k}+13
$$

are $(k, y)=(1, \pm 5),(2, \pm 7),(3, \pm 11)$.

6. Remarks. The fields $Q(\theta), Q(\phi)$, although having the same discriminant $-3^{5} \cdot 13$, are nonisomorphic. In fact, there are precisely three cubic extensions of $Q$ with this discriminant, the third generated by a root $\psi$ of $x^{3}-9 x+24=0$. For, using Hasse [2], we see that if $K$ is any such field, then $K(\sqrt{-39})$ is a cyclic cubic extension of $Q(\sqrt{-39})$ with conductor 9. Since the 3-Ringklassengruppe with conductor 9 in $Q(\sqrt{-39})$ is a product of 2 cyclic groups of order 3 , the corresponding classfield has exactly 4 cubic subfields, each with a conductor (which has to be a rational integer) dividing 9. Similarly, the 3-Ringklassengruppe of conductor 3 has order 3, and so precisely one of these fields has conductor 3. (Note that $Q(\sqrt{-39})$ has class number 4 , so none of the fields has conductor equal to 1.)

It only remains to verify that the fields $Q(\theta), Q(\phi), Q(\psi)$ are nonisomorphic. This may be seen from the fact that the rational prime 5 splits in $Q(\theta)$ but not in $Q(\phi)$, and that 2 splits in $Q(\psi)$ but not in either of $Q(\theta), Q(\phi)$. (In fact, 2 is an inessential discriminant divisor in $Q(\psi)$.)

The above also shows that $Q(\lambda)$ is the unique cubic field of discriminant $-3^{3} \cdot 13$.

Emmanuel College

Cambridge CB2 3AP. England

Department of Mathematics 253-37

California Institute of Technology

Pasadena. California 91125

1. A. Bremner, R. Calderbank, P. Hanlon, P. Morton \& J. Wolfskill, "Two-weight ternary codes and the equation $y^{2}=4 \cdot 3^{\alpha}+13, " J$. Number Theory. (To appear.)

2. H. HASSE, "Arithmetische Theoric der kubischen Zahlkörper auf klassenkörpertheoretischer Grundlage," Math. 7.., v. 31, 1930, pp. 565-582.

3. D. J. Lewis, Diophantine Equations: p-Adic Methods, Math. Assoc. Amer. Studies in Math., Vol 6. 1969. pp. 25-75.

4. E. S. Selmer. “Tables for the purely cubic field $k(\sqrt[3]{m}), "$ Avh. Norske Vid.-Akad. Oslo I, no. 5, 1955.

5. Тн. Sкоц.ем. Ein Verfahren zur Behandlung gewisser exponentialer Gleichunger, 8de Skad. mat. Kongr. Forh. Stockholm. 1934.

6. J. V. USPENSKY. "A method for finding units in cubic orders of a negative discriminant," Trans. Amer. Math. Soc.. v. 33, 1931, pp. 1-22.

7. E. T. Vvanesov, "On a question of a certain theorem of Skolem," Akad. Nauk Armjan. SSR, Ser. Mat. ․ 3, 1968. pp. 160-165. 